\title{
A Ray Tracing Model for Wireless Communications
}

\author{
https://doi.org/10.3991/ijim.v13i11.1173 \\ Hasanain Abbas Hasan Al-Behadili ( ${ }^{\varpi}$ ), Mohsin Najim Sarayyih Almaliki \\ University of Misan, Amara, Iraq \\ dr-hasanain@uomisan.edu.iq \\ Saddam K. Alwan AlWane \\ University of Technology, Baghdad, Iraq
}

\begin{abstract}
Recently, there is an increasing interest in modelling of communication processes. One of the most important reasons for developing such models is to forecast what will happen. A number of modelling studies provide several assumptions and use different theories. But up to now, there is still a lack of an optimized model that can be used for the purpose of future communications. This indicates a need to better understand the aspects of communication modelling and the motivation behind improving our knowledge in this field. This paper details work done to design a propagation model of a wireless communication system to give a forecast of its signal behavior. Data for this study were collected using a wireless communication system employed in the outdoor environment. The final part of this paper gives a summary and description of findings, which include a comparison between the experimental and simulation results. The model finally shows a similar trend with the observation.
\end{abstract}

Keywords-Modelling, Indoor environment, Outdoor environment, ray tracing.

\section{Introduction}

The modelling of signal propagation is essential to designing a robust communication network. The performance of the modelling process depends on the way in which the model has been constructed, such as its empirical and theoretical assumptions. In all cases, the various propagation issues need to be taken into account to make the process is as reliable as possible. These include reflection, refraction, scattering, diffraction and ducting. Researches on communications engineering can be classified in five categories: hardware, measurements, modulation aspects, propagation and networking issues. With regards to this paper, there is an interest in studying of signal propagation. Since the communication systems consist of transmitter and receiver and channel, this paper concerned, mostly, with the channel. Shannon's theory is one of the most famous theorems that deals with the channel capacity in communication systems [1]. Since then, there is still a interest in the modelling of communication process, particularly, modelling of the communication channel. The aim of modelling 
is to provide a suitable representation of any real system for the purpose of planning, which can save time, cost and reduce the complexity in reality.

In this paper, a model of wireless terrestrial propagation was developed. The model consists of different steps employed by the model using MATLAB. The simulated signal path starts from transmission points, then passing through the propagation medium until reaching the receiver. A ray-tracing method has been developed in to deal with the signal path geometry in addition to several considerations to estimate the received signal characteristics. Finally, the output was compared with measurements of wireless signals under different scenarios.

\section{Previous Studies}

A communication model (propagation) can be described as an appropriate mathematical representation of any scheme that can be used as a replacement for any realtime system. Basically, the aim behind the development of modeling is to create a good plan for any real-time process.. An extensive analysis of propagation models can be found in [2]. A survey on simulation of the communication system can be found in [ 3] where there are many aspects have been discussed such as: modelling cosidetions and evaluation of simulation systems. Bcause there are generally two communications environments, e.g. outside and inside strucures, there are two communication models categories; indoor and outdoor. .Outdoor models considered the effects of buildings, trees and other obstacles. Longley- Rice [4], Okamura and Hata models are the most common examples of outdoor propagation models but they differ in frequency coverage.

In indoor propagation models, there are different factors need to be considered such as: the effect of doors and layout of the buildings. Examples of such models are Long-distance path loss and Ericsson Multiple Breakpoint Model and most of these models were evaluated by the International Telecommunication Union [5].

Ray tracing tecniques have been described and evolved to create more realistic models. In ray tracing, the route of signal propagation is traced from the point of departure to the target .Developing such techniques paved the way to design propagation models with more accurate outcomes. For example, a method of wireless communication prediction has been suggested by [6]. The model in this paper was designed for indoor environment to be worked in the UHF band. It developed an improved threedimensional model. In addition to [7] where the effect of shadowing considered in the ray-tracing model which were achieved at $2.4 \mathrm{GHz}$. Additionally, a review for ray tracing models done in [8]. They discussed the challenges and classification of simulation based on ray tracing. They recognize main there categories of ray tracing; Element method, Extended Novel and Progressive. Recent studies have been concerned with using ray tracing in the most dominant technologies i.e. 5G. For instance, [9-10] have employed the ray-tracing model in $5 \mathrm{G}$ network using indoor environment for Millimeter-Wave (used in 5G).

Overall, the above studies demonstrate the importance and usefulness of using the modelling with multiple outcomes in reality .A high level of results will require a 
comparison with measurements which is one of the basic goals of the long term aim of our project and this paper tries to define a model of propagation with a simple example of comparison with experimental data.

\section{$3 \quad$ Methods and Results}

The experimental setup is given in Figure (1). At the transmitter section, there is an RF signal generator which was set to work at $2.4 \mathrm{GHz}$. The generator is followed by a directional antenna which has omnidirectional radiation pattern (as well as the received antenna) and both antennas have vertical polarization. In addition to a terminal linked to the antenna, an oscilloscope and spectrum analyzer were mounted at the receiver side. It is important to mention that there is only one connection between out from the received antenna but for simplicity, it has been used two lines in the figure. However, the spectrum analyser was the only one utilized for the output in this paper and more details about all measurements are ongoing work in this project. While the emphasis on measurements is not the primary subject of this paper, the field of measurements was not very important, but the model must use the same features as the observation when comparing both measurements and modeling.
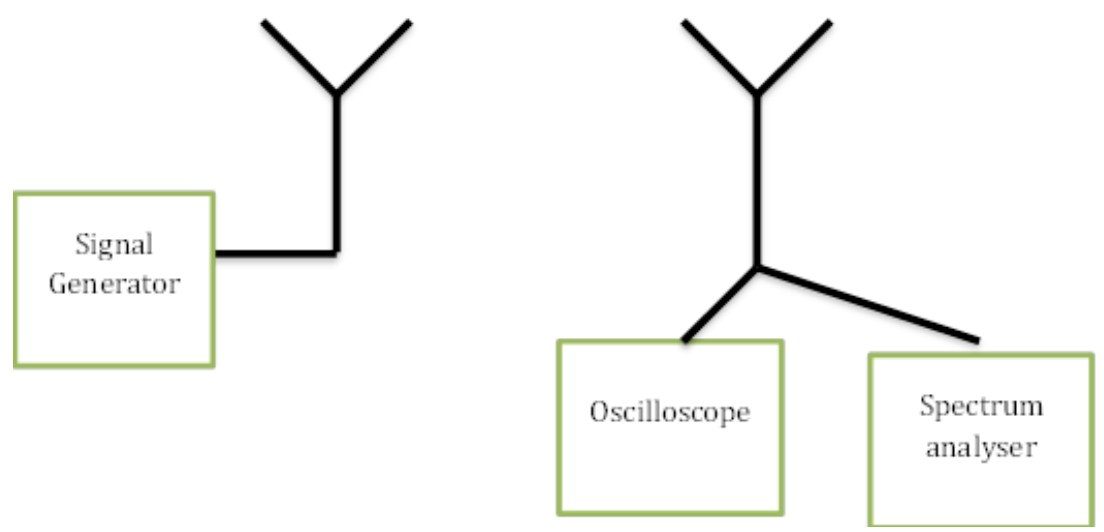

Fig. 1. Experimental work setup

To design a model for a propagation of radio signal, a ray tracing model has been designed by a set of Matlab scripts as shown in Figure 2.

The Algorithm starts with specifying the location of both transmitter and receiver. The location can be given in geographic location and height above the earth. Initially, a calculation of location is being estimated based on Cartesian coordinates, then will be converted into geographic coordinates using a subroutine within the first Matlab scripts given in Figure 2 (LocaLY.m). 


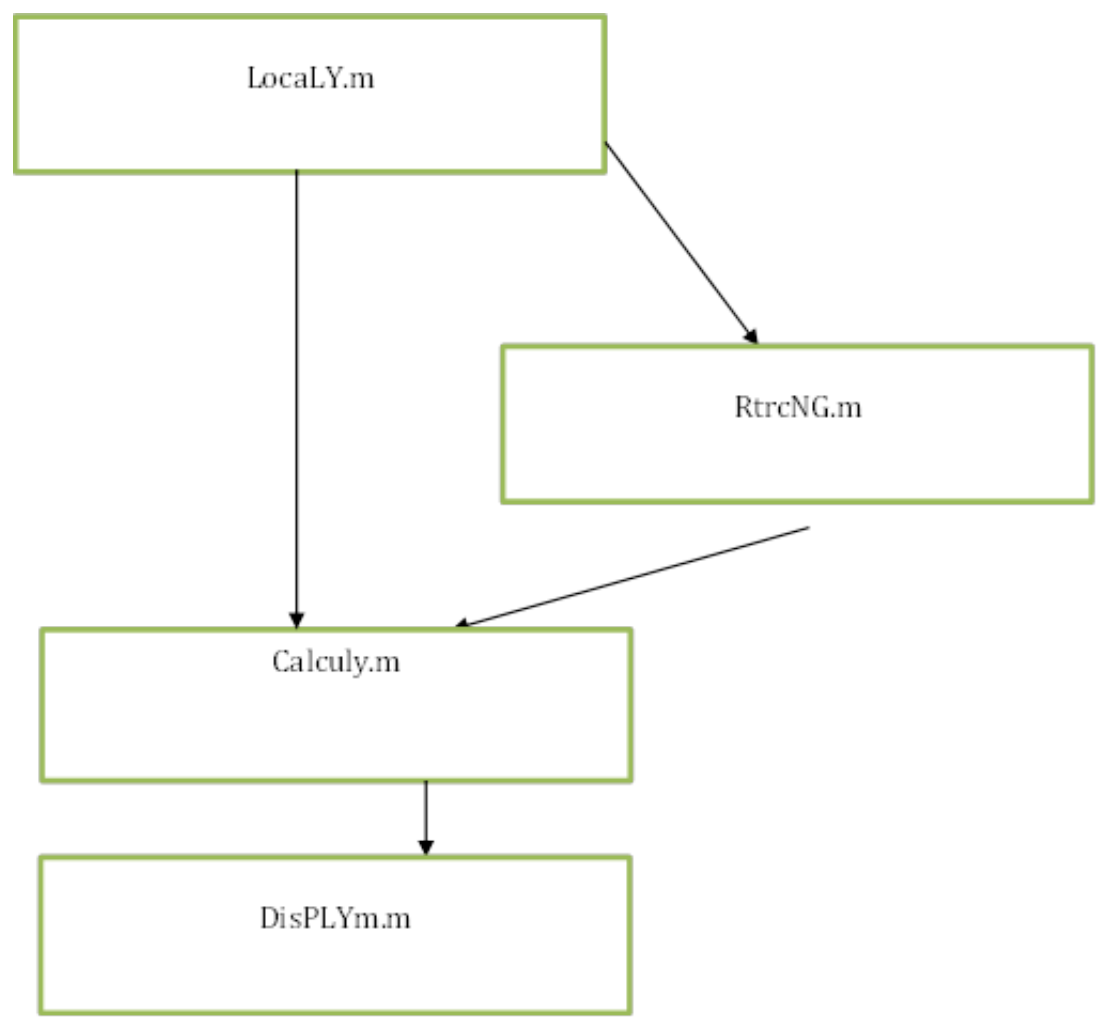

Fig. 2. Matlab scripts used in the model

After that, the signal was propagated through a model of ray-tracing using (RtrcNG.m) script. The system here basically uses a multi-mathematical assumptions propagation model including the two-ray ground model (to substitute the multipath propagation), free-space loss. Besides considering several propagation models such as the model Okumura and the model Hata .The effect of path losses effect was embedded in the subroutines, like fading, interference and Doppler shift effect. It is important to remind here that reflection, diffraction and scattering were followed the basic idea of mathematics for these phenomena. Finally, the ray-tracing model uses geometry to define the positions of the rays comprising the Latitude, Longitude and height.

Next, the signal will trace until it hits the receiver location that has been already defined by the user. Then, the ground geographic location will be re-converted to Cartesian coordinates, to make it easy to define in computer-based work. This will leads to calculating the estimated received power using the following mathematical form:

$$
P_{r}=P_{t} G_{t} G_{r}\left(\frac{c}{4 f \pi d}\right) * A e
$$

where, 
$P_{r}:$ the received power

$P_{t}:$ the transmitted power

$G_{t}:$ the transmitter gain

$G_{r}:$ the receiver gain

c: speed of light

f: frequency of transmission

Ae: the area around the received point.

Ultimately, the Matlab script (DisPLYm.m) was then employed to show the outcomes from the model on demand such as a map of transmitter and receiver locations, the effect of frequency and Doppler. One example is given in figure (3) where a Doppler frequency (in Hertz) is given with regards to the distance between the transmitter and receiver. The modelled Doppler frequency recorded the highest value just below $14 \mathrm{~Hz}$ where it could be as some results of a modelled object gives realistic results. Unfortunately, it was not possible to observe the Doppler frequency and has been postponed to future work.

Another example of the outcomes is shown in Figure (4), which illustrate the received power in $\mathrm{dBm}$ for both empirical observations and modelling. It is expected to show this kind of trend of the signal power distribution between the transmitter and receiver i.e. power goes from higher to lower values as it diverges from the transmitter. Generally, the measured values have perturbed shape as compared with the modelled records but there is a noticeable agreement between both. However, there is a difference between simulation and observation about $8 \mathrm{dBm}$ closer to the transmitter and 45 meters away.

These findings have noticeable implications for the knowledge of wireless signal channel behaviour and good success to provide a model with reliable results. However, there is still a need to do more measurements to compare with the model outcomes in order to test its availability to use.

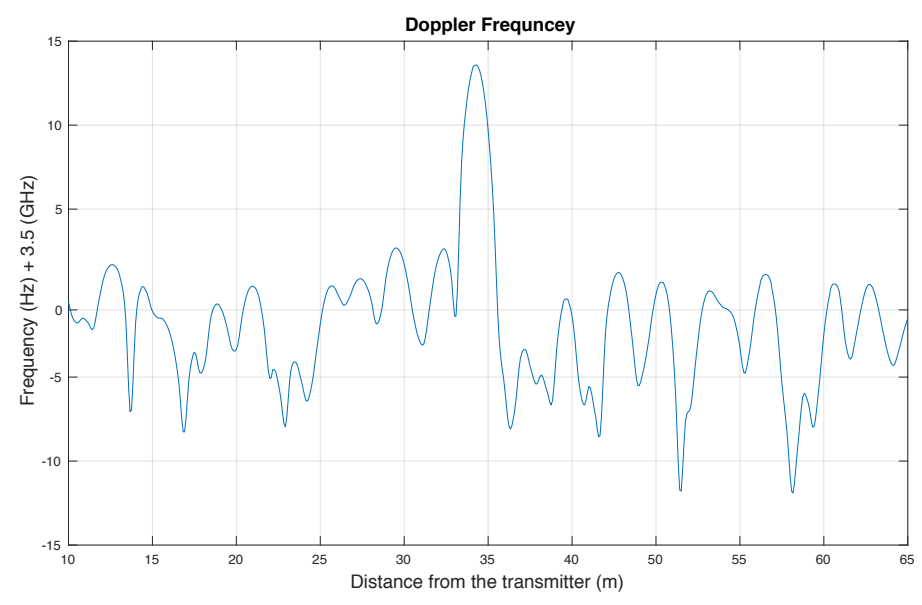

Fig. 3. Simulated Doppler frequency with regards the distance from transmitter 


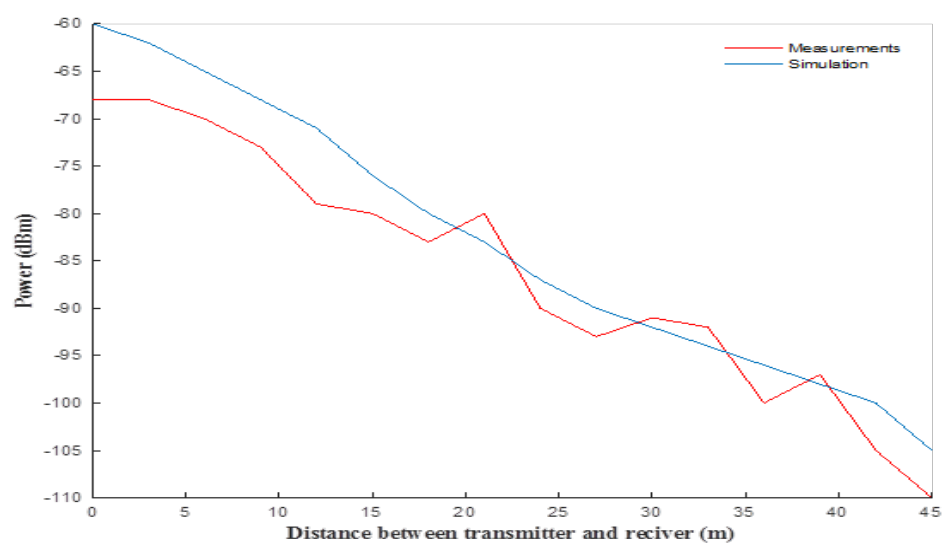

Fig. 4. Received power for measurements and simulations in terms of the distance between transmitter and receiver.

\section{Conclusion}

The current model was designed to introduce a simulation of signal propagation for the frequency that can be used in terrestrial communications. This study has found that there is a possibility to use propagation model for purpose of planning a communication circuit since the results indicate that a model provides close records of signal strength values as compared with observations. These outcomes can be used to help in continuing our projects work to reach the goal. A further study needs to be carried out to investigate the model reliability. Further improvements will suggest the availability to use a ray-tracing model in the $5 \mathrm{G}$ network.

\section{Acknowledgement}

Authors would like to thank Mr. Ameer Al-Shammaa a Ph.D. student at University of Leicester for his help in this paper.

\section{References}

[1] Shannon, C.E., "Communication in the presence of Noise," Proc. IRE, vol.37, no. 1, January 1949, pp. $10-21$

[2] Iskander, M. F., \& Yun, Z. (2002). Propagation prediction models for wireless communication systems. IEEE Transactions on microwave theory and techniques, 50(3), 662-673. https://doi.org/10.1109/22.989951

[3] Jeruchim, M. C. (1995). Modeling and simulation of communication systems: an overview. Journal of the Franklin Institute, 332(5), 521-533. https://doi.org/10.1016/00160032(95)00080-1 
[4] Longley, A. G., \& Rice, P. L. (1968). Prediction of tropospheric radio transmission loss over irregular terrain. A computer method-1968 (No. ITS-67). INSTITUTE FOR TELECOMMUNICATION SCIENCES BOULDER CO.

[5] Series, P. (2012). Propagation data and prediction methods for the planning of indoor radiocommunication systems and radio local area networks in the frequency range $900 \mathrm{MHz}$ to $100 \mathrm{GHz}$. Recommendation ITU-R, 1238-7.

[6] Ji, Z., Li, B. H., Wang, H. X., Chen, H. Y., \& Sarkar, T. K. (2001). Efficient ray-tracing methods for propagation prediction for indoor wireless communications. IEEE Antennas and Propagation Magazine, 43(2), 41-49. https://doi.org/10.1109/74.924603

[7] Jung, J. H., Lee, J., Lee, J. H., Kim, Y. H., \& Kim, S. C. (2014). Ray-tracing-aided modeling of user-shadowing effects in indoor wireless channels. IEEE Transactions on Antennas and Propagation, 62(6), 3412-3416. https://doi.org/10.1109/tap.2014.2313637

[8] Geok, T. K., Hossain, F., Kamaruddin, M. N., Rahman, N. Z. A., Thiagarajah, S., Chiat, A. T. W., \& Liew, C. P. (2018). A Comprehensive Review of Efficient Ray-Tracing Techniques for Wireless Communication. Int. J. Commun. Antenna Propag, 8, 123-136. https:// doi.org/10.15866/irecap.v8i2.13797

[9] Hossain, F., Geok, T., Rahman, T., Hindia, M., Dimyati, K., \& Abdaziz, A. (2018). Indoor Millimeter-Wave Propagation Prediction by Measurement and Ray Tracing Simulation at 38 GHz. Symmetry, 10(10), 464. https://doi.org/10.3390/sym10100464

[10] Hossain, F., Geok, T. K., Rahman, T. A., Hindia, M. N., Dimyati, K., Ahmed, S., \& Ziela, N. (2019). An efficient 3-D ray tracing method: prediction of indoor radio propagation at $28 \mathrm{GHz}$ in 5G network. Electronics, 8(3), 286. https://doi.org/10.3390/electronics80 $\underline{30286}$

\section{Authors}

Dr. Hasanain Abbas Hasan Al-Behadili is a Lecturer at the Department of Electrical Engineering, Faculty of Engineering, Misan University, Iraq. He had a PhD in Communications Engineering from the University of Leicester, UK. He had with him 10 years' experience in teaching Electrical Engineering courses. Dr Al-Behadili has also experience in various programming languages and environments such as $\mathrm{C}, \mathrm{C}++$, Python programming, Matlab and many others. dr-hasanain@uomisan.edu.iq

Mr. Mohsin Najim Sarayyih Almaliki holds a Master's Degree in Computer science by the University of Arts, Science and Technology (AUL), Lebanon. He is currently a Lecturer at the Department of Business Administration, University of Misan, Iraq. He taught several courses since 2013 and published many papers in computer science and communications. Mr Almaliki is keen with different computer languages such as C, C++, Java and others. Muhsen@uomisan.edu.iq

Mr. Saddam K. Alwan AlWane received his Master's degree in Computer Engineering from the University of Technology. He is a Lecturer at the Department of Computer Engineering, University of Technology, Iraq where he thought several courses. alwanesaddam@ymail.com

Article submitted 2019-08-22. Resubmitted 2019-09-28. Final acceptance 2019-09-30. Final version published as submitted by the authors. 